\title{
Suppression of Fault Currents on DG Using Various Fault Current Limiters in Distribution Network
}

\author{
K. Maruliya Begam ${ }^{1 *}$, T. Karthikeyan ${ }^{2}$ and K. Ramani ${ }^{3}$ \\ ${ }^{1}$ Department of EEE, Arulmurugan College of Engineering, Thennilai, Karur, Tamil Nadu, India \\ ${ }^{2}$ Department of Mechanical Engineering, Arulmurugan College of Engineering, Thennilai, Karur, Tamil Nadu, India \\ ${ }^{3}$ Department of EEE, K.S.R College of Technology, Tiruchencode, Erode, Tamil Nadu, India
}

\begin{abstract}
Protection of Distributed Generation (DG) is a challenging task in entire electrical family. DG plays a vital role in advanced power systems engineering. The connection of DG to the distribution network has numerous advantages. Even though it has a advantages, while connecting the DG with existing power system network, the fault current level in many places have often exceeded which causes the abnormal conditions in the entire power system network. In order to reduce the fault current level, various Fault Current Limiters (FCL) are proposed. In this paper hybrid Fault Current Limiter (HFCL) and its applications are proposed to minimize the effect of the DG on the distribution protection system in a radial system during a fault. The simulated results are analysed and compared with a Solid State Fault Current Limiter (SSFCL) and Superconducting Fault Current Limiter (SFCL) using MATLAB simulation. The effectiveness of proposed fault current limiters are determined and examined in the test system.
\end{abstract}

Keywords: Distributed generation; Solid State Fault Current Limiter; Superconducting Fault Current Limiter; Hybrid Fault Current Limiter; Distribution protection system

\section{Introduction}

Nowadays electric utilities try to search new technologies to improve power quality and reliability of their customers. Then distributed generations are becoming attractive to many utilities. Moreover, these technology produce electrical energy with less environmental impacts, their installation are easy, and are highly efficient. Previous studies have shown that distributed generation causes several challenges to the protection of distribution system [1-3]. The main disadvantage of DG is producing fault current that are more than breaking capacity of circuit breakers and fuses. Furthermore, the fault current produced by DG are causing problems in protection coordination, false tripping of feeders and relay mal operation. Several ideas have been introduced as possible solutions [4,5]. These papers propose switchgears and protection coordination upgrade in systems consist of DG. Although these solutions may technically operate, they are complicated and expensive. So, these solutions are not practical for existing distribution systems. A novel idea exists to limit the fault currents produced by DG and prevent switchgears upgrade is FCLs application [6]. The implementation of FCLs in electric power systems is not restricted to suppress the amplitudes of the short circuits; they are also applied in variety of performances such as the power system transient stability enhancement, power quality improvement, reliability improvement, increasing transfer capacity of system equipments and inrush current limitation in transformers [7-10]. Different configurations of fault current limit are proposed to mitigate the impact of DG on distribution protection system [6].

\section{Existing Method}

In order to reduce the fault current level following fault current limiters were used:

\section{Solid State Fault Current Limiter (SSFCL)}

The most beneficial property of this configuration is simplicity of structure and control, low steady state impedance, fast response and high impedance fault. Figure 1 shows a general configuration of the solid-state current limiter. It consists of a fast solid state switch, current limiting impedance, voltage limiting element and series mechanical switch. [10]. The GTO thyristors are used as the fast solid state switch and the current limiting impedance is connected in parallel with these. The GTO thyristors are used to interrupt a current instantly upon receiving a turn-off signal and the current limiter impedance is used as way for passing the fault current when the solid state switch interrupt a fault current. But a sudden interruption of current is likely to cause an overvoltage in the circuit, so the voltage limiting element is used to prevent this [9]. The overcurrent detector and control device

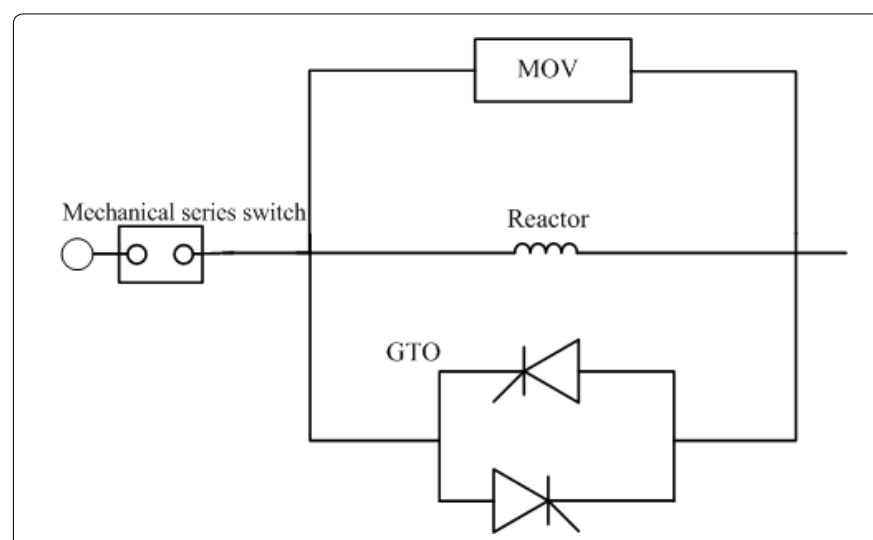

Figure 1: Basic configuration of SSFCL (MOV: Metal Oxide Varistor).

*Corresponding author: K. Maruliya Begam, Department of EEE, Arulmurugan College of Engineering, Thennilai, Karur, Tamil Nadu, India, Tel: 9788518536; E-mail: maruliyabegam@gmail.com

Received December 03, 2013; Accepted January 20, 2014; Published January 22, 2014

Citation: Maruliya Begam K, Karthikeyan T, Ramani K (2014) Suppression of Fault Currents on DG Using Various Fault Current Limiters in Distribution Network. J Electr Electron Syst 2: 115. doi:10.4172/2332-0796.1000115

Copyright: () 2014 Maruliya Begam K, et al. This is an open-access article distributed under the terms of the Creative Commons Attribution License, which permits unrestricted use, distribution, and reproduction in any medium, provided the original author and source are credited. 


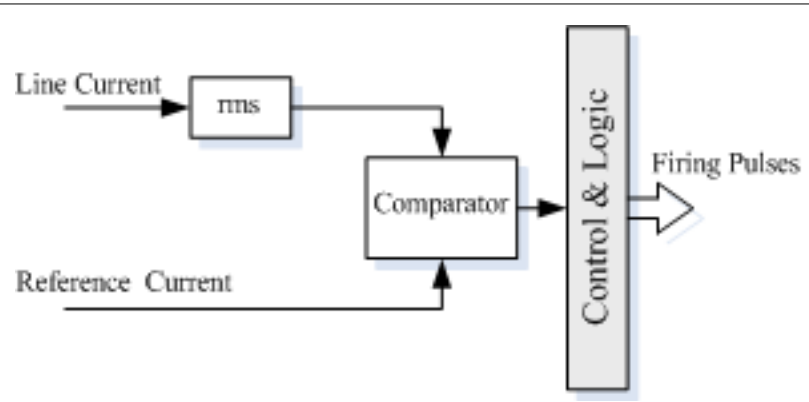

Figure 2: Schematic diagram of SSFCL control.

detect a fault and produce turn-off and turn-on signals for the GTO thyristor. Figure 2 shows the control block diagram of this FCL. Figure 2 is a schematic diagram of solid state FCL control [10]. In steady state condition, the GTOs are gated continuously. When a fault occurs a fault current must be detected rapidly. This is done by comparing the instantaneous current level with a predetermined value. After detecting fault occurred, the turn-off signal is given to the GTO. In this method, it is important to discriminate fault current from an in-rush current. This problem was solved by choosing a suitable detection level. The current limiter must continue to limit the current during fault and returns it to the normal condition automatically after the fault is cleared. Therefore, current magnitude has to be monitored all the time. When detector recognizes the current magnitude small for some period, clearance is assumed and turn-on signals are given to the GTO. Therefore, in steady state operation only switching loss appears which is very smaller than other losses in the network.

\section{Superconducting Fault Current Limiter (SFCL)}

Superconducting Fault Current Limiter (SFCL) is innovative electric equipment which has the capability to reduce fault current level within the first cycle of fault current [2]. The first-cycle suppression of fault current by a SFCL results in an increased transient stability of the power system carrying higher power with greater stability [3]. The most important physical property dominating the current limiting behavior of the SFCL is the electric field current density characteristics of High Temperature Superconductors (HTS) [6] which is dependent on temperatures. Fault current, also called short-circuit current (Isc), describes current flow during a short. It passes through all components in the affected circuit. Fault current is generally very large and, therefore, hazardous. Only the combined impedance of the object responsible for the short, the wire, and the transformer limits its magnitude. One objective of electrical distribution system design is to minimize the effect of a fault, i.e. its extent and duration, on the uninterrupted part of the system. Coordinating the sizes of circuit breakers and fuses assures that these devices isolate only the affected circuits. It prevents a short at an outlet from shutting down power to the entire building. The concept of using the superconductors to carry electric power and to limit peak currents has been around since the discovery of superconductors and the realization that they possess highly non-linear properties. More specifically, the current limiting behaviour depends on their nonlinear response to temperature, current and magnetic field variations. Increasing any of these three parameters can cause a transition between the superconducting and the normal conducting region. The current increase can cause a section of superconductor to become so resistive that the heat generated cannot be removed locally. This excess heat is transferred along the conductor, causing the temperature of adjacent sections to increase. The combined current and temperature can cause these regions to become normal and also generate heat. The term " quench " is commonly used to describe the propagation of the normal zone through a superconductor. Once initiated, the quench process is often rapid and uncontrolled. Though once initiated the quench process is un controlled, the extent of the normal region and the temperature rise in the materials can be predicted. Thus, the quench process can be used in the design into a superconducting component.

\section{Working of SFCL}

The working of SFCL mainly depends upon the impedance of the passing current. A larger transformer can be used to meet increased demand on a bus without breaker upgrades. A large, low impedance transformer can be used to maintain voltage regulation at the new power level. Reduced fault current flows in the high voltage circuit that feeds the transformer, which minimizes the voltage dip on the upstream high voltage bus during a fault on the medium voltage bus.

- When operated below critical parameters, a. Tc (temperature), b. Ic (current), c. Hc (magnetic field).

- Superconductors have virtually zero resistance.

- When operated above Tc, Ic, Hc normal state resistance is restored.

- The inherent ability to "switch" from virtually zero resistance to a finite value when Ic is exceeded can be used to limit shortcircuit fault currents.

$R$-SFCLR-SFCLs utilize the superconducting material as the main current carrying conductor under normal grid operation. The principle of their operation is to suppress the fault current within the first cycle of fault current. It consists of a shunt resistor and variable resistor. The current passing through the shunt resistor is denoted as Ishunt. At present, for HTS materials, the convention is to define "critical current" as the current at which a voltage drop of $1.0 \mathrm{iV} / \mathrm{cm}$ is observed along the conductor. An R-SFCL directly exploits the transition from superconducting to normal state that a material exhibits when the transport current exceeds the critical value.

\section{Proposed Method}

\section{Hybrid Fault Current Limiter (HFCL)}

Due to the use of solid-state devices, solid-state FCL has switching loss. Mechanical breakers and switches exist that have virtually no closed circuit losses, but they cannot operate quickly and are therefore unable to commutate the voltage across their contacts. Thus, a novel hybrid FCL is proposed to solve these problems. During the steady-state operation of the system, the mechanical switch is passing the current with no loss and during fault condition the solid-state switch operates to commutate and solve the arcing problem [7]. The circuit of hybrid FCL is shown in Figure 3. The hybrid FCL consists of three parallel paths. Path A includes a fast mechanical switch which commutates the current onto path B which includes power semiconductor elements. This path forces the current onto resistor element in path $\mathrm{C}$ for fault current limitation. At prefault condition, the fast mechanical switch of path $\mathrm{A}$ is closed and the GTO switches of path B are gated on. Since the resistance of the fast mechanical switch is much smaller than semiconductor switches, almost all of the current flows through path A. When a fault occurs, it takes $40 \mathrm{~ms}$ to detect the fault by fault detection unit. After that a signal is given to the fast mechanical switch in path A to open. The fast mechanical switch opens within $150 \mathrm{~ms}$ and produces an arc voltage drop of about $40 \mathrm{~V}$. The voltage drop across the semiconductor 


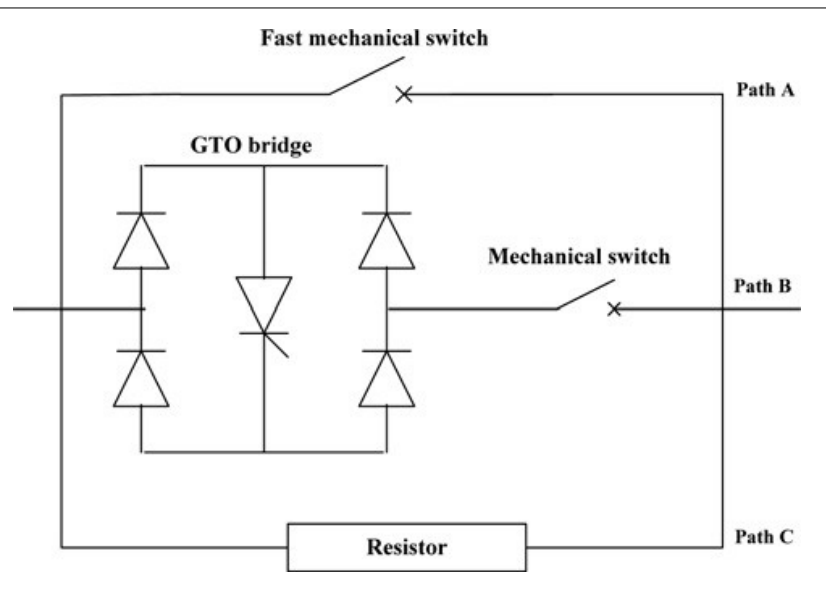

Figure 3: Basic configuration of HFCL.

switches is typically $10-15 \mathrm{~V}$, which is lower than arc voltage; therefore the current starts to commutate from path A onto path B [10]. When the commutation is completely done, the GTO switch is turned off after $150 \mathrm{~ms}$ to transfer the current to path C. The fault current is limited by flowing through the impedance of path $\mathrm{C}$. After that to isolate the semiconductor switches from the high transient recovery voltage the mechanical switch in path $B$ is opened when the current reaches zero. To consider the actual model of FCL, the self inductance in loop A-B and $\mathrm{B}-\mathrm{C}$ is modelled with two inductors $\mathrm{L} 1$ and $\mathrm{L} 2$. To ensure complete current commutation within a short time interval, the self inductance of the loop A-B must be sufficiently low on the order of L1 1/4 $0.5 \mathrm{mH}$. Also, turning off the GTO causes a very high (di/dt), and thus, an excessive voltage rise due to the self-inductance of the loop $\mathrm{B}-\mathrm{C}$ on the order of $\mathrm{L} 2 \mathrm{1} / 410 \mathrm{mH}$. This high rise of voltage is limited by metal-oxide varistor which is used in parallel with GTO bridge. R_FCL is assumed to be $15 \mathrm{~V}$ to limit the value of fault current to the level of load current value. DGs also show transient behaviour during fault condition. The type of DG in this paper is assumed to be synchronous generator. The injected fault current from these DGs, have three states: sub-transient, transient and steady state [10]. Asynchronous machine is considered as conventional power plant. A three phase load is connected to the power plant. Fault occurred here is three phase to ground fault. Here the FCL model developed for single phase is converted to three phases and attached as a subsystem in the distribution line. The three phase to ground fault is induced in both the simulink models. A step input signal is given to the fault block in order to give the specific time of mentioning at what time the fault should occur and which time it should be recovered. The subsystem should operate with the time delay specified in the FCL parameters.

\section{Results and Discussion}

The simulation results show the validity and effectiveness of suggested scheme and also the ability of the HFCL to reduce the fault current is increased. The fault current limitation in the single phase and three phase systems of imposing an upper limit on the current that may be delivered to a load with the purpose of protecting the circuit generating or transmitting the current from harmful effects due to a short circuit in the load. During the presence of FCL the fault current is limited closer to its rated triggering current value. In the single phase system the rated current value is given as 400A. Without FCL the single phase system with fault,the current is above 440A. With HFCL the single phase fault current is limited in the range of 400-410A.In the three phase system the rated current value is given a1800A. Without

\begin{tabular}{|c|c|c|c|c|}
\hline \multirow{2}{*}{ Type of system } & $\begin{array}{c}\text { Current values } \\
\text { during the } \\
\text { absence of FCL }\end{array}$ & \multicolumn{3}{|c|}{ Current values during the presence } \\
\cline { 3 - 5 } of FCL \\
\hline Single phase & 490 & SSFCL & SFCL & HFCL \\
\hline Three phase & $2500-3000$ & $2100-2300$ & $1900-2000$ & $1800-1900$ \\
\hline
\end{tabular}

Table 1: Comparison of current values of single phase and three phase systems with and without FCL.

FCL the single phase system with fault, the current is above 1800$1900 \mathrm{~A}$. With SFCL the single phase fault current is limited in the range of 1900-2000A. The comparison results are shown in Table 1.

\section{Conclusion}

The above tests conducted using the SFCL Single phase prototype showed the excellent current limiting capability of the fault current. It also provides fault current limiting actions very fast. It reduces effectively the short circuit currents to much smaller current amplitudes .Results of simulations are very satisfactory and fully describe the behavior of HFCL devices for all nominal and limiting conditions. These results are very important for studying the behavior and to evaluate the impact of FCL devices on transmission lines and many electrical apparatus networks and also to give useful hints to the design of practical SFCL devices. Thus the HFCL is promising novel electric equipment to reduce excessive fault current in electric power systems effectively. In practical application of this novel device into electrical networks, it causes favourable impacts on the electric power system.

\section{References}

1. Doyle MT (2002) Reviewing the Impacts of Distributed Generation on Distribution System Protection. Power Engineering Society Summer Meeting 1: 103-105.

2. Kauhaniemi K, Kumpulainen L (2004) Impact of distributed generation on the protection of distribution networks. $8^{\text {th }}$ IEE International Conference on Developments in Power System Protection 1: 315-318.

3. Girgis A, Brahma S (2001) Effect of Distributed Generation on Protective Device Coordination in Distribution System. Large Engineering Systems Conference on Power Engineering 115-119.

4. Brahma SM, Girgis AA (2004) Development of adaptive protection scheme for distribution systems with high penetration of distributed generation. IEEE Transactions on Power Delivery 19: 56-63.

5. Brahma SM, Girgis AA (2002) Microprocessor - based reclosing to coordinate fuse and recloser in a system with high penetration of distributed generation. IEEE Power Engineering Society Winter Meeting 1: 453-458.

6. Ye L, Lin LZ, Juengst KP (2002) Application Studies of Superconducting Fault Current Limiters in Electric Power Systems. IEEE Transactions on Applied Superconductivity 12: 900-903.

7. IEEE Standard Inverse-Time Characteristic Equations for Overcurrent Relays IEEE Standard C37.112-1996, September 1996.

8. Ueda T (1993) Solid-state Current Limiter for Power Distribution System. IEEE Transaction on power Delivery 8: 1796-1801.

9. Chabanloo RM, Abyaneh HA, Agheli A, Rastegar H (2011) Overcurrent relays coordination considering transient behaviour of fault current limiter and distributed generation in distribution power network. IET Generation, Transmission \& Distribution 5: 903-911.

10. Shahriari SAA, Abapour M, Yazdian A, Haghifam MR (2010) Minimizing the Impact of Distributed Generation on Distribution Protection System by Solid State Fault Current Limiter. IEEE PES on Transmission and Distribution Conference and Exposition 1-7. 